\title{
EL ESTADO, EL DIÁLOGO Y LA TRANSFORMACIÓN DE LOS CONFLICTOS SOCIOAMBIENTALES
}

\author{
Augusto Castro \\ Instituto de Ciencias de la Naturaleza, \\ Territorio y Energías Renovables de la \\ Pontificia Universidad Católica del Perú
}

\begin{abstract}
Resumen: El artículo plantea los vínculos necesarios que deben existir entre el Estado y el diálogo. Se propone que el Estado es el espacio de diálogo por excelencia y para ello el texto se apoya en la naturaleza ética que es inherente a la constitución del Estado. El Estado, además, representa una perspectiva estratégica y de futuro para la vida humana en el mundo que se expresa y funda en asuntos tan importantes como la igualdad, el reconocimiento y la democracia. Esta reflexión del Estado tiene como interés recolocar el rol del Estado frente a sus dificultades y límites concretos por convertirse en un verdadero espacio de diálogo. El artículo revisa las características que todo diálogo debe tener teniendo en cuenta las características de los conflictos, y en especial de los sociales y ambientales, y a su proceso de su transformación. Se insiste en el papel de apertura, de comunicación abierta y de negociación permanente que se debe tener para enfrentar los desafíos que plantea la sociedad y el medio ambiente. El artículo finalmente, busca revisar ideas y conceptos vinculados al dialogo, busca redefinir criterios y plantear la necesidad de construir capacidades y políticas públicas para enfrentar adecuadamente los desafíos y conflictos que se presentan.
\end{abstract}

Palabras claves: Estado, ética, bioética, diálogo, conflictos, conflictos socioambientales, transformación de conflictos, negociación, políticas públicas. 


\title{
The State, the Dialogue and the Transformation Process of Socio-environmental Conflicts
}

\begin{abstract}
This article discusses the necessary linkages that must exist between the State and the dialogue. To propose that the State is the space for dialogue par excellence, the text relies on the ethical nature that is inherent in the constitution of the State. Furthermore, the State represents a strategic and forward-looking perspective for human life in the world that is expressed and founded on such important issues as equality, recognition and democracy. This reflection about the State aims to reposition the role of the State in the face of its actual difficulties and limitations at becoming a true space for dialogue. The article reviews the characteristics that any dialogue must have taking into account the characteristics of conflicts, and especially socio-environmental ones, and their transformation process. It insists on the role of openness, open communication and permanent negotiation that the State must play to face the challenges posed by society and the environment. Finally, the article seeks to review ideas and concepts related to dialogue, redefine criteria and raise the need to build capacities and public policies to adequately face the challenges and conflicts that may arise.
\end{abstract}

Keywords: State, Ethics, Bioethics, Dialogue, Conflicts, SocioEnvironmental Conflicts, Conflict Transformation, Negotiation, Public Policies. 


\section{Augusto Castro Carpio}

Doctor en Estudios Latinoamericanos por la Universidad de Tokio, Japón. Magíster en Filosofía, Licenciado en Filosofía y Bachiller en Humanidades con mención en Filosofía por la Pontificia Universidad Católica del Perú. Profesor Principal del Departamento de Ciencias Sociales de la PUCP. Ex director del Centro de Investigaciones Sociológicas, Económicas, Políticas y Antropológicas de la PUCP (CISEPA-PUCP). Ex presidente del Comité de Ética para la Investigación con Seres Humanos y Animales de la PUCP. Coordinador de la Red Latinoamericana de Estudios de Sociedad y Ambiente de CLACSO. Fue director del Instituto de Ciencias de la Naturaleza, Territorio y Energías Renovables de la PUCP (INTE-PUCP). Actualmente es presidente del Comité Editorial y coordinador del grupo de investigación Ética, Ambiente y Sociedad del INTE-PUCP.

Entre sus principales publicaciones están: El desafío de un pensar diferente: pensamiento, sociedad y naturaleza (2018); Río + 20 Desafíos y perspectivas (2015); Reconstruir y educar: tareas de la Nación. 1885-1905, y Una educación para re-crear el país. 1905-1930, v.7 y v.8 de la Colección Pensamiento Educativo Peruano (2013); La responsabilidad social empresarial: reflexiones sobre los planes estratégicos de socios estratégicos. Caso Antamina (2012); Los nuevos retos de la política social en el Perú: articulando la academia con la gestión pública (2011), La filosofía entre nosotros: cinco siglos de filosofía en el Perú (2009); El desafío de las diferencias: reflexiones sobre el Estado moderno en el Perú (2008); entre otros.

Correo electrónico: acastro@pucp.pe 


\section{Introducción}

Cada vez que estalla un conflicto, sea social o socioambiental, tenemos la sensación de que varias cosas no se hicieron bien y que probablemente no se tomaron las medidas adecuadas. Pero, ipor qué no se hicieron bien las cosas y no se tomaron las medidas adecuadas? La respuesta a esta pregunta puede llevarnos a determinar que quizá se actuó sin experiencia y perspectiva, o quizá se actuó de mala fe. En ambos casos observamos problemas en el origen del conflicto. En la primera posibilidad, la ruptura de los acuerdos se establece cuando una de las partes considera que no se está cumpliendo con lo estipulado y se siente engañada o dañada; en la segunda situación, una de las partes descubre el engaño y considera que es nulo el acuerdo firmado. En todos estos casos se expresa una falla profunda en el instrumento que debía llegar al acuerdo: el diálogo.

Frente al fracaso de una negociación de carácter nacional la percepción que tenemos es que han fallado las partes; pero debemos decirlo con claridad, quien ha fallado principalmente es el que ha jugado como espacio de diálogo, que no es otro que el Estado. Al no existir un espacio de diálogo el conflicto se agudiza. Nuestra posición está en señalar que el Estado es ese espacio de diálogo. El problema surge cuando el Estado es visto por la ciudadanía como una parte interesada en el conflicto, en defensa de tal o cual interés. ¿Qué es lo que hace que el Estado abandone su papel y tome partido por alguna de las partes? Este ha sido el motivo que nos ha llevado a reflexionar sobre este tema.

En este texto nos interesa sostener que la mejor evidencia de que el Estado es un instrumento de justicia y de derecho es la de, parafraseando a Hegel $(1971,1972)$, reconocerlo como el espacio de diálogo por excelencia. Esto quiere decir que la sociedad arriesga mucho con el papel que se le asigna al Estado. En un Estado que no asume su papel de articulador, de concertador, de espacio privilegiado, el diálogo está condenado al fracaso y con ello se deterioran las condiciones de vida de sus ciudadanos, de su sociedad y de su territorio.

Una observación que se nos puede hacer, y con justicia, es la de que el Estado real y concreto no es precisamente un espacio de concertación, sino que siempre ha tomado partido y 
muchas veces por los más fuertes, como es el caso de las empresas, y que sería inadecuado señalar que el Estado es el horizonte ético o de diálogo privilegiado cuando los ejemplos de su arbitrariedad en la vida social se multiplican. Precisamente este es el tema de discusión y de debate. Nuestra propuesta lo que busca es colocar al Estado en su lugar y desligarlo de su papel instrumental y burocrático que la realidad moderna y el sentido común le han asignado. No hubiéramos intentado reflexionar sobre el tema si no estuviéramos convencidos, ciertamente, de que la ubicación y la práctica concreta del Estado muchas veces están sesgadas por los intereses que lo dirigen.

Conviene señalar, antes de desarrollar nuestras ideas, una crítica a la visión que plantea que el Estado no es más que una representación de clase. Hablar del Estado como representación de clase resalta los vínculos de los intereses económicos y sociales de algunas clases sociales con el Estado, lo cual tiene lógica y es razonable, pero no necesariamente todo criterio lógico se expresa con la misma claridad en la realidad. Pudo haber sido razonable entenderlo así en el siglo XIX, pero hoy en día, al estudiar con detenimiento al Estado moderno, apreciamos que la representación de intereses en el Estado es sumamente compleja y decir que representa sí y solo sí los intereses de algunas clases, puede ser forzado. Hay una relación obvia de las clases con el Estado, pero hay que ser muy preciso cuando queremos determinar con exactitud esa representación y esos vínculos.

No obstante, conviene preguntarse ¿qué es lo que está en la base de esta mirada del Estado que lo entiende solo como un espacio de canalización de intereses particulares? Vayamos por partes.

Si se parte justamente de que el Estado obedece a poderosos intereses de clase y de que por ello no hay Estado de justicia, ni de derecho, ni de diálogo - y podríamos sumar que tampoco hay democracia-, la reflexión que nos viene a la mente es que no habría nada que plantear, nada que hacer y que la única tarea sería volver a pensar el tema de la revolución. Pero el problema es que la revolución no resuelve los conflictos sociales o políticos, como lo hemos podido observar durante los siglos XIX, XX y XXI. Entonces, ¿qué debemos hacer?

En estos momentos no hay espacio para la implementación de un planteamiento de corte revolucionario porque se privilegia la democracia como la manera de cambiar y renovar las cosas y las sociedades. Sin embargo, algunos piensan que las reivindicaciones y las reformas tienen que ser llevadas de manera profunda en las actuales circunstancias; para ello, la exigencia de radicalidad y de movilización son las tareas del momento. Pero existen, frente a una demanda maximalista, solo alternativas minimalistas. Existe un desfase entre las aspiraciones y las propuestas concretas. Esto afecta, sin duda, a la temática del diálogo. 
Dado que el Estado no puede resolver los conflictos porque estos no se resuelven, sino que se transforman, como veremos más adelante, su descrédito es cada vez mayor. Esto ha llevado a la idea de que el Estado es solo el instrumento práctico llamado a resolverlos. Esta idea, hija de la modernidad positivista, es la que queremos combatir en estas líneas. Esa postura cree que los conflictos y los problemas se pueden resolver de una vez y para siempre.

No resulta lejano a esta lógica el que se piense que para encarar estos desafíos estratégicos se necesita un verdadero instrumento real y pragmático que los enfrente. Para este punto de vista, la perspectiva de diálogo no es el instrumento y su posición aparece muy disminuida; al equipararlo con el lenguaje común, se lo considera inútil para llegar a acuerdos que serían necesarios para enfrentar los problemas de fondo. Esta perspectiva no aprecia la importancia de la conversación entre las personas. No solo no comprende la importancia de la sola comunicación para generar alternativas, sino que desconfía de la relación entre la gente. Incluso, no aprecia la llamada Teoría de la acción comunicativa, por poner un ejemplo, que justamente es contrafáctica, es decir que se basa principalmente en la capacidad de argumentación - principios de fundamentabilidad y susceptibilidad de crítica habermasianos con los que cuentan todos los seres humanos- y no solo en la acción propiamente dicha (Habermas 1987). No creer que la gente se comunique y converse, que hable, que parlamente y exponga sus criterios, ideas y sentimientos es negar no solo la base de cualquier diálogo democrático, sino de la condición humana. ¿Acaso el llamado parlamento no tiene en este elemental sentido común su punto fuerte y decisivo?

Sostener la idea del Estado como espacio privilegiado de diálogo es volver a plantear los temas claves de lo que este significa. Todos los poderes en los que se basa el Estado moderno tienen su fundamento en la igualdad, en el reconocimiento y en la democracia. Todos estos instrumentos son construidos desde los ciudadanos y su relación, es decir, desde su práctica dialógica. Ello es el punto más remarcable en la comprensión del sentido ético que el Estado representa.

\section{EL ESTADO COMO HORIZONTE ÉTICO POR EXCELENCIA}

Reafirmar que la ética está en el centro de la práctica del Estado y que este está articulado al llamado «espacio público» nos parece sustancial para recuperar el papel del Estado y del diálogo en un momento en que muchas veces no aparece una mirada comprensiva sobre ellos. Volver a pensar los conceptos nos parece una buena práctica que ayuda a la acción y, en este caso, a la transformación de los conflictos. Es por ello que la capacidad de 
diálogo, y además de mediación que debe tener el Estado en relación con los conflictos, nace precisamente por esta orientación ética que está en su definición (Hegel 1971, 1972).

\subsection{La dimensión ética del Estado}

La ética, como se sabe, es una preocupación por la vida buena tanto privada como pública. Entraña también una preocupación por la conducta, tanto por la de «no hacer daño» (Epicuro 1973) como por lo de «hacer lo que corresponde» (MacIntyre 1982). La dimensión ética atañe a todos los públicos, tanto a la conducta pública como a la privada. En este sentido, el vínculo entre ética y Estado es profundo.

El Estado cumple un papel ético fundamental en la vida de los ciudadanos. Debemos recordar que el Estado nace recogiendo los intereses de cada persona de la comunidad y busca representarlos. No hay ninguna duda en pensar que el papel del Estado está articulado de manera directa con el ejercicio de la democracia y de la justicia (Locke 1997, Rawls 2000). En ambos casos conviene resaltar que la relación entre conducta ética y el papel del Estado están estrechamente relacionados. Incluso muchos autores desde los tiempos antiguos y desde los modernos han afirmado que la plasmación de la conducta ética se expresó en el Estado. Por ello, la preocupación permanente por la democracia, por la igualdad y por la justicia es intrínseca al Estado moderno.

La lógica del Estado está nutrida de la capacidad para el diálogo de sus ciudadanos y este, como veremos más adelante, no solo puede ser visto como un instrumento de privados, sino como la herramienta con la que cuenta el Estado para dirimir los conflictos y problemas de su sociedad.

No puede quedarnos ninguna duda de que el diálogo es la forma más adecuada para procesar y desarrollar estas orientaciones, pero, en realidad, habría que ir más allá: el espacio público que representa el Estado es el espacio privilegiado de diálogo que hace posible la democracia, la justicia y, finalmente, la igualdad.

\subsection{La reflexión sobre la ética para con la naturaleza: la bioética}

Años después de finalizada la Segunda Guerra Mundial surge un nuevo concepto que busca ampliar la perspectiva ética al vincularla con los temas de la naturaleza y de la vida. Nos estamos refiriendo a la bioética. 
Las atrocidades que se hicieron durante esta guerra marcaron para siempre los límites por los que nunca se debería transitar en materia científica y el Código de Núremberg lo pudo expresar con claridad. La ética colocaba sus ojos ya no solo en las relaciones entre ciudadanos y en su capacidad de diálogo y política, sino en una nueva dimensión. Se advertía una nueva perspectiva que exigía colocar los límites entre las personas, la naturaleza y la vida. Al inicio, la bioética abarcó temas de respeto a la vida en seres humanos y tuvo una negación rotunda a que se realicen experimentos científicos en ellos; luego se fue ampliando a una mirada que tomó en cuenta a todos los seres vivos, incluyendo a la naturaleza misma.

La discusión y las exigencias de colocar límites al comportamiento humano frente a la vida de los seres vivos y a la misma naturaleza (Brundtland 1987) plantearon nuevos temas y reflexiones al discurso de la bioética: uno de los puntos de debate, por ejemplo, fue que la relación entre vida sensitiva y vida inteligente, es decir entre vida humana y vida animal, podía formularse mejor. ¿Por qué la importancia de discutir la cuestión de la inteligencia y reeditar este viejo debate? Simplemente porque ello puede ayudar a responder la pregunta de fondo: ¿hasta qué punto los animales pueden ser sujetos morales? (Wall 2014, 2016; Singer 2018). Quizá lo que está aquí en debate es si la inteligencia determina el comportamiento moral y cómo queda todo esto cuando los científicos nos señalan hoy que los animales son seres inteligentes (Young 2017, Lenoir 2018, Diski 2017). Finalmente, los seres humanos son seres morales porque el asunto de la moral está fundado en el juicio racional (MacIntyre 2001).

El tema, sin embargo, ha ido mucho más allá. Hoy hablamos de una relación conflictiva entre la especie humana y la naturaleza, y no se puede negar que el uso de la fuerza y de la tecnología ha infligido golpes severísimos a muchos de los ecosistemas del mundo. Incluso, con la generación de gases de efecto invernadero la humanidad ha puesto en crisis el planeta. Pero esta relación conflictiva con la naturaleza puede poner en riesgo y generar un colapso en la sociedad humana, como lo está mostrando la actual pandemia del COVID-19 que se habría transmitido — según señalan- a través de los animales.

Toda esta situación ha colocado en un nuevo nivel la discusión sobre la ética y, en especial, sobre la bioética. El hecho de contar con inteligencia y tener capacidad moral no le otorga a la especie humana el derecho de destruir el planeta ni de decidir la vida de los otros seres vivos. El poner límite a una práctica humana desbocada en los tiempos modernos frente a la naturaleza, los seres vivos y a los propios seres humanos se vuelve un imperativo moral, y esta tarea de colocar límites es la tarea de la bioética en la actualidad.

Urge, a criterio nuestro, una nueva mirada y una nueva relación con la naturaleza, entendiéndola como una realidad viva con la que se interactúa 
permanentemente. Esta nueva relación puede mejorar a los ecosistemas y al planeta $y$, naturalmente, mejorar la vida entre los miembros de la especie humana.

\section{EL ESTADO Y LA CONSTRUCCIÓN DE FUTURO}

En este punto nos interesa desarrollar, en primer lugar, el papel programático del Estado como visión estratégica que orienta la vida, el espacio territorial y el curso de la sociedad ${ }^{1}$; y, en segundo lugar, que frente a las dificultades y a los conflictos sociales y socioambientales, el Estado debe contar con criterios que le permitan transformarlos y superarlos.

Nuestro interés en este texto, como lo vamos expresando, es vincular el papel del Estado a la cuestión del diálogo como parte inherente de su actividad. Si apreciamos la responsabilidad del Estado en el mundo de hoy, veremos que los temas se han complejizado de una manera impresionante. Si conocemos las dificultades que tenemos entre los seres humanos para llegar a acuerdos, deberíamos preguntarnos ¿con qué criterios vamos a plantear la igualdad - base del diálogo - con los otros seres vivos, los ecosistemas y, finalmente, el planeta? ¿Cómo reconocerlos y ponerse en su condición? ¿Cómo tomar decisiones junto al planeta y los animales, o en función del planeta y los animales? El Estado, como vamos observando, tiene un papel central como espacio ético y de diálogo para tratar los conflictos sociales y también los conflictos socioambientales, donde están implicadas la naturaleza y las diversas formas de vida.

\subsection{Contar con una visión estratégica sobre la sociedad y el mundo}

El Estado se caracteriza o se debe caracterizar por contar con una visión estratégica acerca del futuro. ¿Qué significa futuro? El futuro significa tomar decisiones para el porvenir del mundo y de la sociedad. La sostenibilidad, lo hemos señalado antes (Castro 2018), significa la lucha por la perdurabilidad de la vida, es decir, la lucha porque la vida en el mundo no desaparezca. Esta visión, que es estratégica por su naturaleza, se deberá materializar en

\footnotetext{
1 Confundir la noción de Estado con el aparato burocrático que llamamos Estado es una grave confusión teórica. Decir que el Estado es la representación de la sociedad está bastante lejos de confundirlo con el sistema de administración pública de una nación, una región o un distrito. Si la reforma del Estado fuera solo la modificación de normas de operación del sistema, estaríamos reduciendo su perspectiva profundamente. La reforma del Estado se establece por la reforma de la ciudadanía y ello es desde siempre un problema de ética.
} 
acuerdos políticos que permitan la organización del territorio y la sociedad hacia objetivos que permitan la sobrevivencia de la especie, de todos los seres vivos y del mundo entero.

Sabemos que la toma de decisiones exige claridad sobre cómo debe manejarse la relación entre los seres humanos y entre estos y la naturaleza. Por ello se hace necesaria una mirada integral de los seres humanos y la naturaleza. Teniendo claro un acuerdo sobre estos objetivos estratégicos se podrán construir los instrumentos y las políticas públicas que ayuden al desarrollo de la vida, y, naturalmente, esto permitirá la transformación de los conflictos sociales y ambientales.

Esta perspectiva obliga a ponerse de acuerdo con los objetivos del desarrollo sostenible globales y en cada país. Este esfuerzo en ponerse de acuerdo exige, como es obvio, una amplia capacidad de diálogo $\mathrm{y}$ concertación. Exige también acordar el camino a seguir y las formas prácticas como se deberán ir conquistando los objetivos del desarrollo sostenible. Desde esta orientación, la mirada estrecha y el inmediatismo deben ser superados porque a lo único que pueden aspirar es a soluciones transitorias e intrascendentes.

\subsection{La visión estratégica del Estado: igualdad y reconocimiento de la naturaleza}

Nos parece conveniente que se tome una posición frente a la práctica cerradamente antropocéntrica que ha caracterizado la relación entre el ser humano y la naturaleza. Planteamos este punto porque la relación que se da entre el ser humano y la naturaleza no es de igualdad. Para el ser humano que se afirma en un antropocentrismo duro, la naturaleza no es más que un recurso natural que puede ser usado con arbitrariedad por la especie humana. La dificultad que encontramos es que algunos pueden argüir que no se dialoga con un recurso natural y eso complica la argumentación (Castro 2017).

Lo que podría superar esta relación de verdadera desigualdad es el reconocimiento de que el ser humano no ha dejado, en ningún momento, de ser también él naturaleza. Es decir que hay un punto de igualdad que hermana al ser humano y a la naturaleza, y esa es su condición natural. Este punto le exige al ser humano - definido por él mismo como naturaleza consciente o inteligente - tratar a la naturaleza respetando sus propias características, sus diferencias y su diversidad. Sabemos, además, que la naturaleza humana no podría sobrevivir si la naturaleza es destruida. La naturaleza humana no es un recurso, más bien es la fuente de la vida humana y de la vida de todos los seres. 
La mirada estratégica del Estado debe desarrollar propuestas programáticas y políticas específicas para construir una nueva relación de reconocimiento y articulación entre el ser humano y la naturaleza. El conflicto socioambiental pone en entredicho la vida, tanto de los seres humanos como la de los ecosistemas, porque coloca por encima el interés económico de algunos sobre los intereses de la vida. La economía debe estar articulada a su función básica: permitir el desarrollo de la población y la salud de los ecosistemas, y buscar el equilibrio entre la actividad humana y la actividad natural. Probablemente será necesario empezar a pensar en soluciones basadas en la naturaleza.

Un tema importante que merece ser señalado en este punto es que los conflictos socioambientales se encuentran siempre alrededor de los recursos de agua y de tierra y que, sin duda, su mal uso o su depredación o contaminación incrementarán la pobreza de las personas y de sus comunidades. Estos llamados recursos naturales están en la mayoría de los casos integrados a la cultura de los pueblos y no solo en el Perú, sino en todo el mundo. La afirmación de la preservación y conservación de las fuentes y recursos que la naturaleza provee al ser humano ha sido integrada en las culturas humanas desde tiempos remotos. En esos tiempos le era fácil al ser humano comprender su relación con el mundo y con los sistemas, y sentirse parte del cosmos por su cercanía permanente a la naturaleza. Esto se ha perdido en las sociedades modernas, donde el peso del ser humano y sus tecnologías han pasado a ser centrales y gravitantes y han opacado e invisibilizado la relación humana con el mundo natural.

Reconocer y fortalecer las particularidades y la idiosincrasia de cada población es algo necesario y fundamental porque ayudará a comprender mejor a la naturaleza y permitirá enfrentar mejor los nuevos desafíos. En este caso, es vital que el Estado reconozca: la identidad étnica y la cultura de los pueblos, sus prácticas éticas y morales, así como su sabiduría ancestral, porque en ellas se expresa con mucha claridad una mayor comprensión de la relación entre el ser humano y naturaleza. Pero se hace también necesario desenmascarar la idea y la actitud del ser humano moderno que se ha convencido de que no tiene nada que ver con lo natural. El ser humano moderno vive en un entorno artificial que no le permite advertir su relación con el mundo (Habermas 1989, Berman 2008, Bauman 2013). Los desastres ambientales y las pandemias como la que vivimos son recordatorios trágicos que nos dicen que la naturaleza está ahí presente y que los seres humanos son inmensamente vulnerables frente a ella. 


\section{EL DIÁLOGO COMO HERRAMIENTA PRIVILEGIADA DEL ESTADO}

El diálogo es un instrumento privilegiado del Estado porque se funda, a juicio nuestro, en tres características centrales: primero, la igualdad; segundo, el reconocimiento; $\mathrm{y}$, en tercer lugar, el ejercicio democrático. Estos tres elementos hacen que el diálogo tenga éxito en la transformación de un conflicto. Nuestra idea es que el diálogo es uno de los instrumentos más destacables para construir una adecuada y plena vida social (Habermas 1987).

\subsection{Las características del diálogo}

\subsubsection{La importancia de la igualdad}

La primera característica del diálogo, como acabamos de señalar, se basa en la igualdad. La igualdad supone que los miembros de una comunidad tienen los mismos derechos y por ello son iguales. Este es un asunto que se hunde en la historia. Los antiguos griegos hablaron de isonomía, es decir, de igualdad ante la ley (Aristóteles 1972,1973). La desigualdad, por el contrario, significa que las personas, al no ser reconocidas como iguales, no tienen los mismos derechos.

La igualdad no es una abstracción ni un principio teórico. Probablemente sea el motivo central que está a la base de cualquier lucha, revolución, reforma o cambio. La igualdad no es una idea, es, más bien, una práctica permanente y cotidiana de los seres humanos que pugnan por ser iguales.

Esto, que parece sencillo, muchas veces no está integrado ni bien comprendido en la vida de las comunidades. Algunos miembros de estas se sienten y creen que son mejores que otros en razón de su poder, de su dinero, de sus rasgos físicos y de muchas otras cosas. Por otro lado, miembros de la comunidad se sienten menos en razón de las mismas razones. La cuestión de la igualdad no solo se inauguró en el mundo antiguo, sino que es una de las banderas más importantes del mundo moderno y ni qué decir del mundo actual donde las reivindicaciones de igualdad y lucha contra la desigualdad movilizan a millones de personas.

En buena cuenta, en una sociedad donde hay desigualdad el diálogo no puede darse. La asimetría impide un diálogo serio porque los sujetos en el diálogo no están en el mismo nivel (Vigil \& Zariquiey 2003). La igualdad siempre planteó derechos y una relación de equidad ante la ley. Este es un principio que, como decimos, viene desde muy antiguo. El diálogo al que nos 
referimos se asienta en la igualdad. La asimetría conspira contra cualquier forma de diálogo (Garay 2009, Gamboa 2013).

De la misma manera que la igualdad es la base del concepto de ciudadanía, lo es también de lo que significa el diálogo. El que sepamos que hay carencias en la asunción de la ciudadanía por parte de las personas no puede significar que la ciudadanía no sea un objetivo de la comunidad política, así como la debilidad y la asimetría en el diálogo no significan que este no sea fundamental y que no sea siempre necesario.

\subsubsection{La cuestión del reconocimiento}

La segunda característica que hemos señalado como básica para el diálogo es el reconocimiento. Debemos plantear que el diálogo exige reconocimiento de las partes y, naturalmente, el reconocimiento de sus intereses (Honneth 1987). En este punto podemos observar algunos temas relevantes.

Uno de ellos es que se puede realmente intercambiar puntos de vista y llegar a acuerdos solo cuando se considera que la otra parte es igual y tiene los mismos derechos. Pero una cosa es señalar, normar e indicar la igualdad y otra cosa es reconocerla en la vida diaria. Obviamente, el reconocimiento se basa en la igualdad, pero adquiere su sentido pleno cuando en la vida cotidiana se trata al otro como si fuera uno mismo. Finalmente, la expresión coloquial de «ponerse en la situación del otro» o en «los zapatos del otro», así como la de «asumir como propios los intereses del otro», puede dar una idea de lo que realmente plantea el reconocimiento (Kant 1983).

Desde ningún punto de vista el diálogo es un monólogo. En el monólogo no hay comunicación, ni intercambio, ni diálogo posible. El diálogo se construye como tal cuando las personas u organizaciones interactúan, se vinculan realmente entre ellas y, por ende, se reconocen. Lo que hace el diálogo es construir un espacio de relación y convivencia. El reconocimiento no es una idea, sino el espacio de concreción práctica de las relaciones humanas en igualdad. El diálogo se establece siempre en una relación con los otros, es decir, en comunidad.

La teoría del reconocimiento de Axel Honneth plantea justamente una mirada concreta de lo que debe ser la relación entre las personas. La igualdad jurídica, llamada igualdad ante la ley, que ha orientado y normado por siglos la relación de derecho entre los seres humanos, deja hoy en su lugar a la práctica del reconocimiento. El reconocimiento plantea con mucha fuerza precisamente la idea de no quedarse en las abstracciones, sino en operar y materializar la relación entre los seres humanos. La igualdad jurídica viene a ser superada por el reconocimiento. Solo basta nuestra condición humana para ser reconocidos. El objetivo del Estado actual tiene en las exigencias del reconocimiento su más profundo desafío. 


\subsubsection{El sentido de la democracia}

La tercera característica es que el diálogo es una herramienta democrática porque toma siempre en cuenta tanto los intereses como las opiniones - la situación real y la palabra- de todos los participantes.

En este punto ya no se trata de pensar solo en una relación de igualdad ni en el necesario reconocimiento que debemos tener entre todos, sino particularmente en el esfuerzo de tomar decisiones todos juntos. La democracia nos lleva a tomar decisiones y a actuar juntos ante los proyectos que hemos acordado. Como diría Norberto Bobbio $(1990,1997)$, la democracia es el sistema donde todos toman decisiones para todos. Probablemente la palabra clave es todos. En el diálogo, como en el Estado, si no participan todos, si no se toman en cuenta los intereses de todos y si no se delinea un horizonte común, no podemos hablar de democracia. Obviamente, la varilla de la democracia, como la de la igualdad y la del reconocimiento, es muy alta. Esa es la orientación maximalista, pero para construir este horizonte se necesitan pasos y un camino a construir. Esto nos exige contar con los mecanismos necesarios para que se realicen nuestros acuerdos y proyectos. El diálogo democrático nos lleva así, necesariamente, a la gestación de políticas públicas.

Tomamos decisiones juntos y democráticamente porque la sociedad humana tiene que enfrentar desafíos de sobrevivencia y de desarrollo. No es solo un problema de buenos y cordiales sentimientos, sino una necesidad vital para vivir en el mundo. Las decisiones democráticas tienen que ver con la sociedad, pero tiene que ver con la naturaleza y las decisiones que debemos tomar en función de ella.

\subsection{El diálogo como herramienta privilegiada para llegar a acuerdos}

En este horizonte, el diálogo entendido como reconocimiento, basado en la igualdad y como parte del ejercicio democrático, debe construir acuerdos.

¿Qué es un acuerdo? El acuerdo es un pacto que se hace desde el corazón, es decir, desde lo más profundo. Los acuerdos fundantes son los que se hacen desde y con el corazón. Quizá sea bueno recordar que la palabra acordar viene del latín accordare, cordis, que significa 'corazón'. La reflexión que podemos hacer al respecto es que no se trata de cualquier acuerdo, sino de uno que compromete profunda e integralmente a quien lo lleve adelante y que la exigencia de vivir juntos lo pone sobre el tapete (Rousseau 1982). 
El acuerdo es un compromiso que se adquiere para enfrentar en conjunto los desafíos de la vida. Sale desde lo más profundo de nuestro corazón, pero se compromete necesariamente con objetivos comunes y prácticos para el futuro de la vida social.

En este punto debemos plantear algunos elementos que nos parecen centrales: uno, el de comprender el diálogo como fuente inagotable de acuerdos, y el otro, el de la posibilidad de transformar los conflictos por medio del diálogo.

\subsubsection{El diálogo como fuente inagotable para llegar a acuerdos}

Debemos plantear, de acuerdo a la experiencia, que la posibilidad del diálogo jamás se agota y que siempre es posible llegar a acuerdos y buscar alternativas. Puede parecer una actitud porfiada el pensar que el diálogo puede ser permanente. Pero lo que estamos sosteniendo es la capacidad de buscar acuerdos y superar los conflictos. Si de verdad se quiere salir de un conflicto, no habrá razón o dificultad que lo impida.

No hay conflicto, por grande y grave que sea, que no pueda ser enfrentado, conversado, debatido y transformado, si hay efectivamente la voluntad de diálogo de las partes que se encuentran en conflicto.

Queda claro que la negación al diálogo expresa con toda nitidez la voluntad del más fuerte o la del que piensa que con su poder puede solucionar el problema. Es el caso, por ejemplo, de extremar la negociación, mentir o cerrarse a negociar o a conversar, que son simplemente expresiones de no querer dialogar ni enfrentar el conflicto. El objetivo de esta negativa y de este proceder casi siempre es buscar doblegar la voluntad del otro sobre la base de la fuerza, el engaño y el poder. Estamos, en este caso, ante una conducta que utiliza el diálogo para sus fines y que en sentido estricto no lo asume con sinceridad.

\subsubsection{El diálogo es siempre expresión de buena voluntad}

Al contrario de imponer la fuerza o el poder, la verdadera actitud del diálogo es la buena voluntad para llegar a acuerdos. Esto indica con claridad cuál es el horizonte desde donde se va a dialogar. Si alguien piensa que en el diálogo puede conseguir y obtener a su favor todos los temas en disputa o en debate, está realmente equivocado.

Los que utilizan el diálogo como una trampa o una estrategia para imponer su voluntad, olvidan que la lógica del diálogo es precisamente lo contrario, es permitir, más bien, que todos ganen y que nadie pierda, porque en el debate ambas partes saben que pueden ceder y ambas partes saben 
qué alternativas tienen para ofrecer y a la vez qué se puede lograr. Nadie puede ingresar al diálogo pensando que va a ganar todo y que no va a perder nada. El diálogo no es una partida de cartas donde uno puede llevarse todo el pozo. En el diálogo nadie gana todo, porque el llamado pozo - es decir, la ganancia- es salir del conflicto - que es el verdadero pozo- y no el de hacerse con todos los puntos de la negociación.

Lo nuevo es que con el diálogo estamos ante la realidad de contar con una nueva oportunidad para nuestros objetivos, porque el conflicto significó que estos ya no se podrían obtener y se habrían cerrado nuestras opciones. Con el diálogo nuestros objetivos pueden ser repensados y probablemente conseguidos. La buena voluntad es un tema importante porque expresa la posibilidad de que el diálogo llegue a buen puerto. Debe quedar meridianamente claro que no contar con una buena voluntad en el diálogo es el camino más seguro al fracaso y a la intensificación del conflicto. No hay manera de hacer las cosas bien si seguimos la lógica del maquiavelismo de interés propio o la del que piensa como Hobbes, que los acuerdos sin espada no son acuerdos (Maquiavelo 1971, Hobbes 1984, Sorel 1976). Parece que algunos en la modernidad han querido construir sus acuerdos políticos sobre la mala voluntad.

\subsection{El diálogo como herramienta básica para transformar un conflicto}

Sostenemos, por todo lo expuesto hasta aquí, que el diálogo apunta a la transformación del conflicto. Hemos pensado muchas veces que los conflictos se resuelven y la realidad nos muestra que la dinámica de los acontecimientos no camina en esta dirección. Ningún conflicto se resuelve de una vez y para siempre. Lo real es que los conflictos se intensifican como se dice, escalan-, es decir, aumentan o se mantienen latentes, pero nunca se resuelven del todo.

La posición que tenemos es que los conflictos no se resuelven en sentido estricto, sino que se transforman. Lo señalamos porque una vez que los conflictos estallan se hace necesario buscar salidas, nuevas opciones y alternativas. Lo real es que, una vez instalado el conflicto, ya no se puede seguir así, y urge un cambio y una transformación.

¿Qué es un conflicto?

En un texto que escribimos en 2018, a propósito del tema, decíamos:

La palabra conflicto viene del latín flígere que significa «chocar, darse un topetón, golpear» (Martínez Guzmán 2005). De ahí el sentido de afligir, 'sentirse golpeado', y de infligir, que significa 'dar un golpe a alguien'. 
El prefijo co en la palabra conflicto significa 'compañía, participación o cooperación'; lo que lleva a la idea de que chocamos entre nosotros, que nos infligimos golpes, y a la vez que, nos sentimos afligidos por los golpes que nos hemos dado. Ciertamente que en la etimología de la palabra conflicto se alude a una relación de tensión y de enfrentamiento entre las partes. Esta idea, que parte de la etimología, nos ayudará a pensar mejor en la noción de conflicto. (Castro 2018: 307-308)

La etimología de la palabra conflicto se refiere a enfrentamiento. Esa idea básica no la podemos dejar pasar porque expresa una realidad permanente de la vida social y de la vida en relación con la naturaleza. Los conflictos son parte de la vida.

\subsection{1 ¿Solución o transformación en el caso de los conflictos?}

¿Por qué se dice que los conflictos no se resuelven? En términos generales, los conflictos sociales y en especial los socioambientales son fenómenos complejos y expresan historia y una diversidad de intereses y problemas. Un conflicto cualquiera puede parecer algo que conceptualmente se puede resolver muy rápido. Quizá formular o pensar en una alternativa puede ser relativamente fácil, pero concretarla y ejecutar una salida práctica puede ser muy difícil, además de tomar tiempo.

Quizá alguien puede suponer que hay conflictos que se han resuelto. Pero esto no deja de ser una mirada ligera, superficial, en suma, un espejismo. Lo más probable es que los conflictos hayan mutado, su tensión haya disminuido y su agresividad haya pasado, pero lo real es que la mayoría de las veces se mantienen latentes y vuelven a activarse y a estallar en el momento menos pensado. Debemos saber que la base de todo esto está en las desigualdades que expresa la sociedad actual ${ }^{2}$.

Si afirmamos que no hay victoria absoluta y radical de una parte sobre la otra, es porque pensamos que las alternativas entrañarán siempre una negociación.

El pensar que tanto los conflictos sociales como los socioambientales se solucionan de una vez y para siempre es seguir pensando con la lógica del que considera que puede ganar todo en una negociación y en un espacio de diálogo, como hemos ya señalado. Los conflictos no se resuelven de una vez ni se solucionan de manera definitiva. Por ello, la actitud positiva con la que se debe ingresar al diálogo es la del que solo espera dar un paso más en el camino de una nueva alternativa. El camino supone salir

\footnotetext{
2 Si el 1\% de la especie humana hasta el año 2011 es dueño del 46\% del PBI del mundo (PNUD 2016), es decir de la riqueza que existe, comprenderemos que los conflictos van a ser permanentes.
} 
de lo más grave y escabroso del conflicto y avanzar a un terreno que permita nuevos horizontes y perspectivas. Creer que se puede volver a la situación anterior no deja de ser una ingenuidad, tanto porque la historia no se repite, como porque volver atrás significaría regresar a los motivos y prácticas que generaron el conflicto. El diálogo, en el seno del conflicto, nos abre caminos que deben ser trabajados con perseverancia, creatividad e inteligencia.

\subsubsection{La importancia de la negociación permanente}

Como acabamos de plantear, no querer negociar es mantener el conflicto, ya sea por la fuerza o por el poder. El que busca transformar el conflicto comprende que la negociación debe ser permanente.

Pongamos un ejemplo, la situación de pobreza es, sin duda, uno de los más graves problemas que afecta a la población latinoamericana y peruana, y está fundada en un conflicto de intereses que se manifiesta en la desigualdad social y económica de millones de personas. Sabemos que mantener la pobreza, la desigualdad y la exclusión, vale decir el conflicto que privilegia los intereses de los que tienen más sobre los que tienen menos, expresa el poder de los primeros sobre los segundos. ¿Cómo esperar superar este conflicto sin una negociación permanente y a largo plazo? ¿Cómo pretender una solución definitiva si la situación es complicadísima y difícil? La experiencia de la Mesa de Concertación para la Lucha contra la Pobreza en el Perú puede ser un buen ejemplo de ello (Mesa de Concertación 2007). Se trata de un ejemplo que pone en perspectiva la lucha contra la pobreza y piensa que su éxito está a largo plazo y con el concurso de todos los implicados. ¿Qué papel juega el Estado? Juega un papel de articulador y de concertador. Juega finamente el papel de colocarse al mismo nivel de los participantes, lo que nos recuerda el principio de la igualdad; plantea el reconocimiento de todos los implicados; y finalmente, decide democráticamente con todos. Los elementos de igualdad, reconocimiento y democracia no son abstracciones para la Mesa de Concertación para la Lucha contra la Pobreza.

Pongamos otro ejemplo en relación a los conflictos socioambientales. Se pensó durante un buen tiempo que era posible resolverlos con base en la fuerza o en el poder político o económico de unos sobre otros. Temas de agua (Salmón 2014, Castro 2014, Faccendini 2015, Urteaga, Guevara \& Verona 2016), de minería (Castillo 2006, Arellano 2011, Castro 2013, Arce 2015), de tierras y de industrias extractivas como los casos de la Oroya (Arzobispado de Huancayo 2005, Bravo 2015), Tambogrande (Alvarado 2001, Ávila 2002, Rousseau \& Meloche 2002, Bebbington 2007, Alayza 2009) y Bagua (Alza et al. 2013, Cavero 2014, Abanto 2019), por poner algunos ejemplos en el Perú de estos últimos años. Todos demuestran a la saciedad que el pretender dar 
soluciones definitivas a los problemas no solo no los soluciona, sino que los agudiza y los vuelve endémicos y permanentes.

La realidad obliga a que estos conflictos socioambientales lleven a una negociación permanente entre las partes y a la comprensión de que no es posible resolverlos unilateralmente (Sotomayor 2016). Sabemos que hay experiencias positivas en el manejo de los conflictos que han permitido a las industrias y comunidades, y naturalmente al Estado, beneficiarse con las nuevas posibilidades que se abren al transformar un conflicto (Diez 2011). Es el caso, por ejemplo, de Anglo American Quellaveco en Moquegua, una minera que logró la aprobación de su Estudio de Impacto Ambiental en 2000 y en 2010, y en las negociaciones que se realizaron con comunidades por el tema del agua y que duraron dieciocho meses hasta 2012. No obstante, siempre hay negociaciones y conversaciones, y el diálogo deberá estar siempre abierto ante cualquier eventualidad porque, como hemos señalado, los conflictos se transforman permanentemente (PNUD-Gobierno Regional de Moquegua 2014).

El diálogo es el único camino para enfrentar estos problemas y nos permite considerar la inmensa complejidad de los asuntos que se encuentran insertos en el conflicto. No buscar salidas y alternativas a los conflictos sociales como la pobreza y a los conflictos socioambientales como los que hemos mencionado a modo de ejemplo, es decir, no saber negociar en pro de un verdadero acuerdo social y económico, y no ser perseverante y creativo en ello, significa simplemente no querer transformar la desigualdad en igualdad, negar el reconocimiento a las partes y mantener el conflicto en una posición de fuerza y de poder que no plantea ninguna salida.

\subsection{El Estado como el espacio de diálogo para transformar los conflictos}

En este apartado final nos gustaría reflexionar sobre la importancia de renovar nuestra manera de pensar al enfrentar en términos prácticos el conflicto y, en segundo lugar, reafirmar la importancia de la formulación y ejecución de políticas públicas como forma de materializar la transformación de los conflictos.

\subsubsection{Renovar nuestro pensar para enfrentar los conflictos}

Para abordar este tema es conveniente tener en cuenta de manera metodológica tres insumos que nos parecen importantes: una revisión de las ideas y conceptos sobre la materia; un esfuerzo por redefinir criterios; $\mathrm{y}$, finalmente, un intento de construir competencias y capacidades. Hay 
bastante literatura construida sobre el tema y conviene que vayamos revisando el abanico de asuntos que se nos presentan (Castro 2016).

\section{- Revisión de ideas y conceptos sobre el conflicto}

Entre los conceptos a considerar tenemos varios: la idea de resolución, la de arreglo, la de acuerdo y, finalmente, la de paz. Nos parece importante volver a pensar, a la luz de los acontecimientos actuales, algunas de sus implicancias por el impacto que tienen en el manejo de los conflictos. Todas ellas tienen que ver con las salidas posibles de un conflicto.

La idea de resolución de conflictos tiene severos límites al creer que existen soluciones definitivas a los conflictos. No obstante, recoge la importante motivación de superarlos, pero no se puede pensar y creer que estos van a tener una solución definitiva; la mayoría de las veces las soluciones solo serán pequeños acuerdos que posponen o transforman los problemas. Conviene comprender, como hemos señalado, que el conflicto es inherente a la naturaleza humana y se debe aprender a vivir con los conflictos, y también que soluciones integrales son muy complejas y tardan tiempo.

En cuanto al concepto de arreglo, debemos plantear que la aspiración a un arreglo es uno de los supuestos básicos para querer transformar un conflicto. Un arreglo puede consistir en una manera de posponer el conflicto o también de prevenirlo o transformarlo. Se puede entender como una especie de alto al fuego o también de aceptar que no se puede cambiar nada y que se opta por dejar las cosas como están.

La idea de acuerdo, sobre la cual ya hemos señalado algunas cosas, plantea que ante el posible arreglo y la superación del conflicto se puede hablar de acuerdo entre las partes. Si el arreglo logra detener la parte más aguda y dolorosa del conflicto y se llegan a establecer pactos generales y específicos sobre el conflicto, podemos estar, finalmente, ante un acuerdo. El acuerdo supone pasar a un nuevo nivel de diálogo en el que las condiciones del conflicto han sido superadas y aparece un nuevo escenario para la negociación (Pulgar Vidal 2016).

Finalmente, la idea de paz entendida como concordia y cese de la violencia, y no como la vieja Pax romana que significaba la victoria sobre el vencido, resulta necesaria (Kant 1984, Castro 1993). La concordia, literalmente con corazón, significa la posibilidad de superar verdaderamente el conflicto y pasar de estar en una posición cerrada a estar en una posición de colaboración y desarrollo mutuo. Nos debe quedar claro que la llamada Pax, como sometimiento del vencido, como la victoria que se da con una de las partes que aplasta a la otra, traerá necesariamente de vuelta el conflicto más temprano que tarde. 


\section{- Redefinir criterios fundamentales}

Los criterios básicos a los que nos referimos para abordar los conflictos son centralmente la cuestión de la prevención y la de la negociación y mediación de los mismos. Estos criterios nos pueden ayudar a comprender cómo enfrentar los conflictos de manera más concreta y advertir los errores más comunes que se cometen al enfrentarlos. Se trata de la experiencia en el manejo de los conflictos.

La prevención de conflictos es la capacidad para ponerse por delante de los posibles estallidos de los conflictos. La prevención no solo se adelanta a los conflictos, sino que busca generar apertura y diálogo. Mirar en profundidad y mirar a largo plazo son los requerimientos básicos en este punto (Defensoría 2017).

La prevención de los conflictos sociales y de los conflictos socioambientales exige un manejo general de la problemática social y política. Obviamente se debe contar con una mirada y una visión de futuro, como hemos planteado. Se sabe que los cambios en las políticas económicas, por ejemplo, pueden desatar graves conflictos sociales y sindicales. Asimismo, las políticas de encubrimiento de la corrupción o de los delitos, o de abuso racial o sexual, pueden provocar multitudinarias manifestaciones que generan enfrentamientos de todo tipo. Por eso, mantener políticas de desarrollo integral, regional y de asistencia son muchas veces las garantías para mantener determinados acuerdos sociales y la llamada «paz social». Igualmente, respetar la ley y no encubrir a corruptos, como no permitir prácticas de odio racial o de acoso, pueden ser muy útiles. Habrá que ser lo suficientemente conscientes de que vivimos hoy en un mundo con mucha vulnerabilidad social y política que pone las condiciones para que los conflictos se desarrollen y estallen sin control.

En cuanto a la negociación y mediación, consideramos que representa una capacidad y un manejo muy especializado con el que se debe contar para enfrentar los desafíos que plantean los conflictos. Si se enfrenta una situación complicada, que puede volverse más grave, lo primero que se debe evitar es «poner más leña al fuego». La prudencia, el diálogo, el conocimiento de las partes y del terreno, el saber recoger las diversas opiniones, el reconocer, aceptar y conocer en detalle los diversos intereses en juego, así como los límites que marcan cualquier negociación, son los requisitos para buscar una salida favorable a todas las partes y avanzar hacia una posición menos compleja y conflictiva (Calvo 2014).

El Estado no puede ser parte, es mediación. El Estado no puede ser juez y parte, como lamentablemente muchas veces lo ha hecho, generando una mayor agudización del conflicto. 
La mediación significa que permite y da fuerza a las diversas partes a fin de superar los conflictos. Si el Estado se parcializa con los empresarios o con una de las partes porque se siente presionado, no se podrá tener nunca una salida a los conflictos. Muchas veces se ha visto al Estado vinculado a los intereses de los más fuertes, probablemente pensando que sus rentas dependen de los ingresos de las grandes empresas, pero esto a todas luces es un craso error. No obstante, se ha visto ceder a algunas autoridades en asuntos graves para ganarse votos, dinero o las simpatías del pueblo. Esta situación desigual en la mediación generará una desconfianza inmensa y permanente en el Estado que está llamado a ser una institución de todos los ciudadanos.

\section{- La construcción de capacidades y competencias}

Para un abordaje adecuado de los conflictos es necesario construir capacidades y competencias en la sociedad y en el Estado. Estas capacidades y competencias fortalecen la capacidad de las partes en un conflicto y fortalecen el papel de Estado como mediador entre estos. Las competencias que una sociedad ha alcanzado deben haber sido internalizadas por ella, y se puede decir que representan un sentido común para la acción.

La primera de ellas es la cooperación entre las partes, que pasa a ser un elemento necesario en la construcción y en la transformación de un conflicto. Hablar de diálogo es reconocer que estimamos que la otra parte tiene alguna razón o razones que no podemos negar y que por eso estamos dialogando. No se dialogaría con quien no tenemos nada que conversar, que hablar o que reconocer. Las partes tienen que cooperar para que el acuerdo se produzca.

La otra competencia consiste en el empoderamiento de las partes en disputa, que no significa que se fortalezcan los elementos del conflicto, sino el reconocimiento de las partes que participan y están inmersas en el conflicto. La razón es muy simple, porque cualquier negociación y cualquier diálogo necesitan que las partes sepan exactamente qué es lo que plantean y cuáles son sus requerimientos y sus límites. Conviene que las partes estén en las mismas condiciones de igualdad para que el diálogo se desarrolle y la negociación llegue a buen puerto.

La conciliación de intereses probablemente sea uno de los puntos más peliagudos porque ninguna de las partes está interesada en perder parte de lo que llevó al conflicto. Pero también las partes saben que deben conciliar porque pueden perder todo si no lo hacen, permitiendo así que su posición se debilite. La conciliación debe ser entendida como un sentido común necesario para cualquiera de las partes. Como acabamos de sostener, el esfuerzo para el éxito exige una negociación muy fina y concertada basada en un diálogo muy profundo.

Finalmente, la capacidad del reconocimiento, como ya lo hemos 
señalado anteriormente, implica que las partes se reconozcan como sujetos iguales y legítimos. No habrá ni diálogo ni negociación cuando este principio no esté expresado clara y adecuadamente. Se trata de un reconocimiento de fondo y de forma. Para la negociación esto se expresa en un protocolo particular y específico que coloca a las partes en el mismo pie de igualdad y de negociación. Muchas veces la pérdida de la formalidad ha destruido el diálogo y la negociación, y el conflicto se ha mantenido y se ha agudizado aún más. En este punto conviene acentuar la práctica de un trato justo, igualitario y de reconocimiento tanto racial, como de género o de clase. Si esto se viola no habrá ni arreglo, ni acuerdo y el conflicto pervivirá.

\subsubsection{La formulación y ejecución de políticas públicas para la transformación de los conflictos por parte del Estado}

La transformación de los conflictos significa superar las tensiones que lo originaron y crear nuevos objetivos y escenarios comunes para construir el futuro. Por ello la perspectiva y el horizonte los da el Estado como expresión programática del acuerdo de los ciudadanos. Nada más estrecho que la política de un Estado o de una autoridad que vende su papel por un plato de lentejas, que significan los tributos que puedan pagar los inversionistas por tal o cual operación industrial, o entregar el territorio por la presión o el chantaje de la población a cambio de votos. Reducir el rol del Estado por una prebenda o por clientelaje a lo único que lleva es al desastre.

Es de suma importancia que el Estado asuma su papel como espacio de diálogo y de concertación. La agenda del Estado debe expresar su capacidad tanto de diálogo como de ejecución de políticas de transformación de los conflictos. Esto quiere decir que la formulación de las políticas públicas llevará necesariamente a la transformación de los conflictos; incluso más, las políticas públicas desarrolladas permitirán arribar a un nuevo horizonte y a un nuevo futuro.

Nos encontramos muchas veces con conflictos que enfrentan a grupos de población y que afectan a los ecosistemas (Postigo 2013, Postigo \& Young 2016). Pongamos ejemplos: la desforestación, la destrucción de bosques y pérdida de la biodiversidad; también la mala gestión de los recursos hídricos, y la contaminación de ríos y lagunas por residuos tóxicos o simplemente basura, entre muchos otros. ¿Qué debe hacer el Estado? Siguiendo con estos ejemplos, el Estado buscará construir políticas públicas. En relación a la conservación del bosque, buscará desterrar la tala ilegal e impedir la pérdida de la biodiversidad, definirá así objetivos, metas y actividades en esa orientación (Roca 2015); sobre el manejo del agua, acordará pautas normativas para una gestión adecuada y sostenible de los recursos hídricos y colocará el tema del cuidado del agua como un asunto de la agenda tanto 
local como nacional; finalmente, impedirá la contaminación de ríos y lagunas, y colocará límites, sanciones y multas al vertimiento de tóxicos y de basura en ellos. Como en estos casos y en muchos otros, el papel del Estado es también claramente práctico. Debe responder con propuestas concretas a los problemas y conflictos suscitados.

Finalmente, habrá que remarcar que las políticas públicas no son declaraciones. Esto significa que una política pública establece una orientación clara y determinada que se expresa en una acción concreta y específica del Estado. Por ello conviene reiterar con fuerza que una política pública no es una declaración. El Estado se expresa en políticas públicas específicas que reflejan el diálogo constante, los acuerdos sobre los intereses en pugna, y la capacidad de llevar a la práctica lo establecido y acordado.

Las políticas específicas de la acción del Estado deben tener en cuenta varios temas de orden organizacional específicos. Uno de ellos, y quizá central, es el de contar con una Dirección Nacional de Diálogo como mecanismo de negociación permanente y abierta a todo tipo de iniciativas. No es una oficina más. Es una oficina que está bajo la dirección de la autoridad máxima del Estado, el Jefe de Estado, que es quien convoca al conjunto del Estado y a todos sus poderes. Hay que recordar en estos tiempos de confusión que ni el presidente del Congreso ni el del Poder Judicial son presidentes de la nación. El presidente de la Nación es el responsable del Estado como un espacio privilegiado de diálogo y no lo puede delegar. En el caso peruano, las oficinas de diálogo nacional no han tenido el apoyo de las autoridades del Estado ni han sido dirigidas por sus autoridades máximas, que muchas veces han delegado su poder en funcionarios que, hay que reconocer, con mucha buena voluntad y esfuerzo han hecho todo lo posible pero que muchas veces no han tenido el poder de tomar las decisiones finales. El desdén por el diálogo y la falta de interés por transformar los problemas han creado una serie de conflictos endémicos y la sensación de que las autoridades están de espaldas a los ciudadanos.

El tema del diálogo es un asunto esencialmente político y así debe ser tratado. Nuestro interés es recuperar para el Estado el poder concertador y de diálogo que ha sido capturado por intereses empresariales o populistas que le impiden cumplir con su papel de espacio privilegiado de diálogo.

\section{Referencias}

Abanto, A. (2019). Lo ocurrido en Bagua fue un fracaso para nuestra vida democrática. Entrevista. Noticias Ser, 6-5. Lima Perú.

Alayza Mujica, R. (2009). La convivencia, un problema de todos. A propósito del conflicto de Bagua. Páginas, (215), septiembre, 6-15. 
Alayza Mujica, R. (2016). ¿El Estado en falta? Actores y creencias en las políticas de reconocimiento y justicia en Perú. Ponencia presentada Lassa. Mayo, Lima.

Alvarado, G. (2001). Recursos naturales y políticas públicas en el conflicto agro minería de Tambogrande. Puno: SEPIA.

Alza, C. et al. (2013). Crónica de un conflicto anunciado. El caso del conflicto social en Bagua (5 y 6 de junio de 2009). En Alza, C. (Comp.). Aprender de la experiencia. Ocho estudios de caso para enseñar Políticas Públicas y Gestión Pública (pp. 121-145). Lima: Escuela de Gobierno y Políticas Públicas.

Arce, M. (2015). La extracción de recursos naturales y la protesta social en el Perú. Lima: Fondo Editorial PUCP.

Arellano, J. (2011). ¿Minería sin fronteras? Conflicto y desarrollo en regiones mineras del Perú. Lima: PUCP, IEP, Universidad Ruiz de Montoya.

Arendt, H. (1974). La condición humana. Barcelona-Buenos Aires-México: Paidós. Aristóteles (1972). Ética Nicomaquea. México: UNAM.

Aristóteles (1973). Política. México: Editorial Porrúa. S.A.

Arzobispado de Huancayo (2005). Estudio sobre la contaminación ambiental en los hogares de La Oroya y concepción y sus efectos en la salud de sus residentes. Huancayo: Arzobispado de Huancayo.

Avila, R. (2002). Tambogrande: conflicto socioambiental y participación ciudadana. Páginas, 27(174), abril, 34-41.

Bauman, Z. (2013). Modernidad Líquida. México: Fondo de Cultura Económica.

Bebbington, A. (Ed.) (2007). Minería, movimientos sociales y respuestas campesinas. Lima: IEP, CEPES.

Berman, M. (2008). Todo lo sólido se desvanece en el aire. La experiencia de la modernidad. Madrid: Siglo Veintiuno Editores.

Bobbio, N. (1990). Fundamento y futuro de la democracia. Trad. de Gabriel Del Favero. Valparaíso: EDEVAL.

Bobbio, N. (1997). El futuro de la democracia. Trad. de José F. Fernández Santillan. Segunda reimpresión. México: Fondo de Cultura Económica.

Bravo, F. (2015). El pacto faústico de la Oroya: el derecho a la contaminación «beneficiosa». Cuadernos de Investigación Kawsaypacha 5. Lima: INTEPUCP.

Brundtland, G. H. (1987). Informe sobre la Comisión Mundial sobre el Medio Ambiente y el Desarrollo. Washington, D.C, PNUMA.

Calvo Buendía, E. (2014). La historia de las negociaciones sobre el cambio climático. En La Conferencia sobre Cambio Climático COP 20: Las perspectivas y los temas críticos para el Perú. Lima: IDEI-Konrad Adenahuer Stiftung-SPDA.

Castillo, G. (2006). Se vende oro: La creación de espacios contestados en la promoción de la minería peruana. En Cánepa, G. \& Ulfe, M. E. (eds.). Mirando la esfera pública desde la cultura en el Perú. Lima: CONCYTEC.

Castro, A. (1993). La paz como fundamento del orden político. Revista PAZ, II Época, I (27). Lima: CEAPAZ. 
Castro, A. (2014). Cambio climático, crisis hídrica y derechos de agua. En Guevara Gil, A. \& Verona, A. El derecho frente a la crisis de agua en el Perú. Primeras Jornadas de derecho de aguas. Lima: PUCP-CICAJ.

Castro, A. (2016). Criterios para encarar, transformar y superar los conflictos entre minería y población. En Metas del Perú al Bicentenario. Lima: Consorcio de Universidades.

Castro, A. (2017). Consideraciones éticas para una mirada comprehensiva de la naturaleza. Revista Kawsaypacha: Sociedad y Medio Ambiente, (1), juliodiciembre. Lima: PUCP.

Castro, A. (2018). El desafío de un pensar diferente. Pensamiento, sociedad y naturaleza. BA, Argentina: CLACSO.

Castro, S. (2013). Pobreza, Minería y Conflictos Socioambientales en el Perú. Lima: INTE-PUCP.

Cavero, O. (2014). Después del Baguazo: Informes, diálogo y visiones del conflicto. En Henriquez, N. (coord.). Conflicto social en los Andes: protestas en el Perú y Bolivia (pp. 230-270). Lima: Fondo Editorial de la Pucp.

Código de Núremberg (1947). Recuperado de: http://www.bioeticanet.info/ documentos/Nuremberg.pdf

De la Cuadra, F. (2016). Cambio climático y justicia ambiental. Lo público desde los movimientos sociales, las comunidades. En Lampis, A. Cambio ambiental global, Estado y valor público: La cuestión socio-ecológica en América Latina, entre Justicia Ambiental y «Legítima depredación». Bogotá: Universidad Nacional de Colombia - CLACSO - INTE-PUCP.

Defensoría del Pueblo (2017). El valor del diálogo. Adjuntía para la prevención de conflictos sociales y la gobernabilidad. Serie Documentos Defensoriales, Documento No. 29. Lima.

Diez, A. (2011). ¿Por qué son necesarios un proceso, una ley y un procedimiento de consulta en el Perú? En Ramos, G. Informe Consulta Previa. Derecho Fundamental de los Pueblos Indígenas e Instrumento de Gestión Estatal para el Fortalecimiento de la Democracia (pp. 21-26). Lima: Congreso de la República.

Diski, J. (2017). Lo que no sé de los animales. Bogotá: Seis Barral.

Durand, F. (2017). Los doce apóstoles de la economía peruana. Una mirada social a los grupos de poder limeños y provincianos. Lima: Fondo Editorial PUCP.

Epicuro (1973). Carta a Meneceo. En García Gual, C. \& Acosta Mendez, E. Ética de Epicuro. La génesis de una moral utilitaria. Barcelona: Barral Editores S. A.

Faccendini, A. (2015). La escasez social del agua: cuestión de lesa ambientalidad. La administración pública del bien común y la administración estatal. Rosario: UNR Editora.

Gamboa, A. (2013). Pueblos Indígenas y conflictos socioambientales: Los casos de Camisea, Amarakaeri y Curaray. Lima: Derecho, Ambiente y Recursos Naturales, DAR. 
Garay C. \& Tanaka, M. (2009). Las protestas en el Perú entre 1995- 2006. En Grompone, R. \& Tanaka, M. (Eds.). Entre el crecimiento económico y la insatisfacción social (pp. 59-123). Lima: IEP.

Grupo Temático de Ciencias Sociales-MINSA (2020). Por una nueva convivencia. La sociedad peruana en tiempos del COVID-19. Escenarios, propuestas de política y acción pública. Lima: Fondo Editorial PUCP.

Habermas, J. (1987). Teoría de la Acción Comunicativa. Madrid: Taurus Ediciones.

Habermas, J. (1989). El Discurso Filosófico de la Modernidad. Madrid: Taurus Ediciones.

Hegel, G.W.F (1971). Filosofía de la Historia. Barcelona: Ediciones Zeus.

Hegel, J.G.F, (1972). Fenomenología del Espíritu. La Habana: Editorial de Ciencias Sociales, Instituto Cubano del Libro.

Hobbes, T. (1984). Leviatán o la materia, forma y poder de una república eclesiástica y civil. México: Fondo de Cultura Económica.

Honnet, A. (1987). La lucha por el reconocimiento. Por una gramática moral de los conflictos sociales. Barcelona: Crítica.

Kant, I (1984). La Paz Perpetua. Madrid, Espasa-Calpe, Octava Edición.

Kant, I. (1983). Fundamentación de la metafísica de las costumbres. Madrid: Espasa-Calpe.

Lenoir, F. (2018). Carta abierta a los animales y a los que no se creen superiores a ellos. Bogotá: Ariel.

Locke, J. (1997). Dos ensayos sobre el gobierno civil. Madrid: Espasa.

MacIntyre, A. (1982). Historia de la Ética. Barcelona: Ediciones Paídós.

MacIntyre, A. (2001). Animales racionales y dependientes. Por quélos seres humanos necesitamos virtudes. Barcelona-Buenos Aires-Mexico: Paidós.

Maquiavelo, N. (1971). Obras Políticas. La Habana: Editorial de Ciencias Sociales, Instituto Cubano del Libro.

Martínez Guzmán, V. (2005). Podemos hacer las Paces: reflexiones éticas tras el 11-S y el 11-M. Bilbao: Desclée de Brouwer.

Mesa de Concertación de Lucha contra la Pobreza (2007). Futuro sin Pobreza, Balance de la lucha contra la pobreza y propuestas. Lima: MCLCP.

PCM, Secretaría de Gestión Social y Diálogo (2012-2020). WILLAQNIKI, Reporte de Diferencias, Controversias y Conflictos Sociales.

PNUD (2016) Informe sobre Desarrollo Humano 2016 Desarrollo humano para todas las personas. Nueva York: PNUD.

PNUD-Gobierno Regional de Moquegua (2014). Sistematización de la Mesa de Diálogo de Moquegua. Experiencia de diálogo, transformación de conflictos y construcción de consensos. Lima.

PNUD-PNUMA (2009). La integración de los vínculos entre pobreza y medio ambiente en la planificación nacional de desarrollo: Un manual de buenas prácticas. Número de trabajo: DRC/1084/NA. Producido por el Centro para la Pobreza y Medio Ambiente del PNUD-PNUMA. 
Postigo, J. (Ed.) (2013). Cambio Climático, Movimientos sociales y Políticas Públicas. Una vinculación necesaria. Santiago de Chile: ICAL.

Postigo, J. \& Young, K. (2016). Naturaleza y Sociedad. Perspectivas socio-ecológicas sobre cambios globales en América Latina. Lima: DESCO, IEP e INTE-PUCP.

Pulgar Vidal, M. (2008) La institucionalidad ambiental en el Perú: Ha llegado el tiempo de madurar. En Oxfam. Pobreza, Desigualdad y Desarrollo en el Perú. Informe Anual 2007-2008 (pp. 33-43). Lima: OXFAM Gran Bretaña.

Pulgar Vidal, M. (2016). El Acuerdo de París: El largo proceso hacia el éxito. Rol, Retos y Oportunidades para el Perú. Lima: Ministerio del Ambiente.

Rawls, J. (2000). Teoría de la Justicia. México: Fondo de Cultura Económica.

Roca, F. (2015). Bosques peruanos, servicios ecosistémicos y servicios socioambientales. En Bernex, N. \& Castro, A. Río + 20 Desafíos y Perspectivas. Lima: Fondo Editorial de la Pontificia Universidad Católica del Perú.

Rousseau, J. J. (1982). Contrato Social. México, Espasa Mexicana, S.A.

Rousseau, S. \& Meloche, F. (2002). El oro y la tierra: retos del desarrollo democrático. Informe de la Misión de observación de la consulta vecinal de Tambogrande, Perú. Canadá: Rights \& Democracy.

Salmón, E. (2014). El derecho al agua en el derecho internacional de los derechos humanos. En Guevara Gil, A. \& Verona, A. (2014). El derecho frente a la crisis de agua en el Perú. Primeras Jornadas de derecho de aguas. Lima: PUCP-CICAJ.

Singer, P. (2018). Liberación animal. Barcelona: Taurus.

Sorel, G. (1976). Reflexiones sobre la violencia. Madrid, Alianza Editorial.

Sotomayor, A. (2016). Remediación de pasivos ambientales mineros como estrategia para el cuidado del ambiente. En Metas del Perú al Bicentenario. Lima: Consorcio de Universidades.

Urteaga, P., Guevara, A. \& Verona, A. (2016) El Estado frente a los conflictos por el agua. Terceras Jornadas de Derecho de Aguas. Lima: CICAJ-INTE-PUCP.

Vigil, N. \& Zariquiey, R. (Eds.) (2003). Ciudadanías inconclusas. El ejercicio de los derechos en sociedades asimétricas. Lima: Corporación Alemana al Desarrollo (GTZ)-PUCP.

Wall, F. de (2014). El bonobo y los diez mandamientos. En busca de la ética entre los primates. Barcelona: Tusquets Editores.

Wall, F. de (2016). ¿Tenemos suficiente inteligencia para entender la inteligencia de los animales? Barcelona: Tusquets Editores.

Young, R. (2017). La vida secreta de las vacas. Bogotá: Seix Barral Los tres Mundos. 